•生物编目・

\title{
东北虎豹生物多样性红外相机监测平台概述
}

\author{
王天明 $1,2,3$ \#冯利民 $1,2,3$ \# 杨海涛 $1,2,3$ 鲍 蕾 $1,2,3$ 王红芳 $1,2,3$ 葛剑平1,2,3* \\ 1 (生物多样性与生态工程教育部重点实验室, 北京 100875) \\ 2 (东北虎豹国家公园保护生态学国家林草局重点实验室, 北京 100875) \\ 3 (北京师范大学生命科学学院, 北京 100875)
}

摘要: 东北虎豹生物多样性红外相机监测平台始建于2006年，位于中国东北温带针阔混交林区，覆盖老爷岭、张 广才岭和完达山, 面积达1.5万多平方公里。平台的监测目标是从生态系统水平上对东北虎(Panthera tigris altaica)、 东北豹 (P. pardus orientalis)、有蹄类猎物及同域分布的其他哺乳动物、森林栖息生境、环境要素和人类活动等进 行全面系统的调查和观测。截至2019年6月，平台产生视频记录超过78.5万条，有效相机工作日173.6万多天，记录 了28种野生兽类和32种野生鸟类。另外, 利用红外相机平台已经在野生动物多样性本底调查、虎豹种群分布、数 量与扩散限制、同域食肉动物种间关系、动物生境利用等方面取得一些成果, 同时为东北虎豹国家公园生物多样 性监测、评估和管理提供了科技支撑。

关键词: 东北虎; 东北豹; 物种名录; 红外相机; 生物多样性监测

\section{An introduction to Long-term Tiger-Leopard Observation Network based on camera traps in Northeast China}

\author{
Tianming Wang ${ }^{1,2,3}$, Limin Feng ${ }^{1,2,3}$, Haitao Yang ${ }^{1,2,3}$, Lei Bao ${ }^{1,2,3}$, Hongfang Wang ${ }^{1,2,3}$, Jianping Ge ${ }^{1,2,3}$ * \\ 1 Ministry of Education Key Laboratory for Biodiversity Science and Engineering, Beijing 100875 \\ 2 National Forestry and Grassland Administration Key Laboratory for Conservation Ecology in the Northeast Tiger and \\ Leopard National Park, Beijing 100875 \\ 3 College of Life Sciences, Beijing Normal University, Beijing 100875
}

\begin{abstract}
The Long-term Tiger-Leopard Observation Network (TLON) is a camera trap based program that was established in 2006 by Beijing Normal University. TLON covers an area of more than 15,000 $\mathrm{km}^{2}$ and is located in the temperate broadleaf and mixed forest in Northeast China. This area covers the Laoye Mountains, Zhangguangcai Mountains, and Wanda Mountains. The goals of TLON are to monitor the status of the Amur tiger (Panthera tigris altaica), Amur leopard (Panthera pardus orientalis), ungulate prey, and other sympatric mammal species. Additionally, a goal for TLON is to study animal's response to different environmental factors and human activity. As of June 2019, TLON has more than 785,000 video recordings that include recordings for 28 wild mammal species and 32 wild birds species that span 1,736,000 days of camera trapping. TLON has helped advance several fields of scientific research which include: surveying of wildlife diversity, studying population status of animals, understanding the distribution and threats for tigers and leopards, interactions between sympatric carnivore species, and mammal habitat use. TLON has also helped with monitoring, evaluation, and the management of biodiversity in the Northeast Tiger and Leopard National Park.
\end{abstract}

Key words: Amur tiger; Panthera tigris altaica; Amur leopard; Panthera pardus orientalis; species list; camera trapping; biodiversity monitoring 
能具有极为重要的作用(Sunquist，2010)。由于濒临 绝境, 野生虎和豹的生存和保护在20世纪就已是国 际性议题。对所涉国家而言，保护工作开展得好坏、 研究水平的高低, 也具有重大国际影响(Dinerstein et al, 2007; Walston et al, 2010)。作为亚洲温带针阔 混交林旗舰物种的东北虎(P. t. altaica) 和东北豹 $(P$. p. orientalis)曾广泛分布于我国东北、俄罗斯远东地 区和朝鲜半岛的原始森林 (田瑜等, 2009; Miquelle et al, 2010a)。但在过去的一个世纪里, 东北虎和东 北豹种群与分布范围急剧衰退, 人为猎杀、猎物贵 乏和栖息地丧失是其主要原因(李钟汶等，2009; Miquelle et al, 2010b)。目前, 野生东北虎仅有500只 左右, 主要残存于俄罗斯锡霍特山脉和中俄边境地 Sugimoto et al, 2014; Wang et al, 2017, 2018)。
区, 濒临灭绝(Miquelle et al, 2010a; Wang et al, 2016)。野生东北豹则更加濒危，它远不如野生东北 虎那么受人关注, 长期被忽视甚至被遗忘, 21 世纪 初调查只发现25-35只左右残存于俄罗斯滨海边疆 区西南部不足2,500 $\mathrm{km}^{2}$ 的区域, 随时可能有灭绝风 险(Pikunov et al, 2003; Hebblewhite et al, 2011)。而 且, 由于种群和生境缩小、近交衰退, 这些残存野 生虎豹生存质量仍在持续下降(Henry et al, 2009;

我国东北广衰的温带针阔混交林曾是东北虎、 东北豹的故乡, 是其最主要的历史分布区, 分布面 积曾达到约 30 万平方公里, 占野生虎和豹(以下简 称野生虎豹或虎豹)分布区总面积的60\% (田瑜等, 2009; Miquelle et al, 2010a)。20世纪90年代末期, 野 生虎豹在我国东北基本销声匿迹。21世纪初, 我国 东北中俄边境开始出现野生虎豹活动的相关报道。 然而, 这些报道只是基于零星的观测和短期的调查, 对于我国东北境内是否还有野生虎豹长期活动、野 生虎豹还有无可能在故土重新定居, 缺乏科学回 答。面对生境的丧失和退化, 中国东北虎豹种群恢 复和保护需要精确的生态信息, 然而在中国这两个 大型猫科动物的基础生态学研究非常贵乏。基于此, 北京师范大学在原国家林业局支持下, 与吉林省林 业厅、黑龙江省森工总局和边防部队等单位组成联 合队伍, 经过近15年的努力, 已在我国东北逐步建 立了一个东北虎豹生物多样性红外相机监测平台 (Long-term Tiger-Leopard Observation Network based on camera traps in Northeast China)。

根据我们的长期监测, 当前东北虎豹已在中俄
跨境区域形成种源, 为中国虎豹种群恢复和生态系 统修复提供了重大机遇(肖文宏等, 2014; Wang et al, 2016)。东北虎豹的保护将成为继大熊猫(Ailuropoda melanoleuca)之后中国生物多样性保护的重要标 志。大中型动物物种濒危机制的研究是生物多样性 研究和保护中的重要课题, 也是保护生物学所要解 决的三大迫切问题之一(Kelt et al, 2019)。面对大量 物种灭绝和濒临灭绝这一严峻的现实, 我们对于物 种，尤其是稀有和濒危物种的了解仍然相当贫乏, 这使得现有的物种保护缺乏科学依据, 给物种的保 护和可持续利用带来了困难。东北温带针阔混交林 支撑和维持着独特和多样的野生动物区系, 特别是 濒危的东北虎豹的存在使得该区域成为全球生物 多样性关注和研究的热点区域, 是建立生物多样性 科学研究综合平台的最佳区域，占据着重要的科学 地位。因此，在该区域开展生物多样性监测、物种 濒危机制以及濒危物种种群恢复途径的研究具有 重要的科学价值和现实意义。

\section{1 平台简介、历史和监测目标}

东北虎豹生物多样性红外相机监测平台始建 于2006年，位于黑龙江和吉林两省东部，从中俄边 境线开始，根据东北虎豹向中国可能的扩散路径， 监测区域逐步向中国内陆扩展。经过十几年的发展, 目前该平台覆盖了中国东北5个国家级自然保护区, 13 个林业局，面积约 1.5 万平方公里(图1)。平台的监 测目标是从生态系统水平上对东北虎豹、有蹄类猎 物及同域分布的其他哺乳动物、森林栖息生境、环 境要素和人类活动等进行全面系统的调查和观测, 获取长期和系统性的生态监测数据, 重点开展东北 虎豹等野生动物的种群生态学、行为生态学、繁殖 生物学、景观和保护生态学等领域研究, 建成一个 “动物与植物、宏观与微观、理论与应用”相结合的 生物多样性监测网络, 并成为具有国际重大影响力 的生态学研究平台, 同时为东北虎豹国家公园自然 资源监测、评估和管理提供科技支撑，为我国国家 公园与自然保护地的野生动物等自然资源监测提 供示范。2018年12月，该平台已建设成为国家野外 科学观测研究站。

\section{平台设计与数据库说明}

(1)数据采集。根据东北虎的主要猎物野猪(Sus 


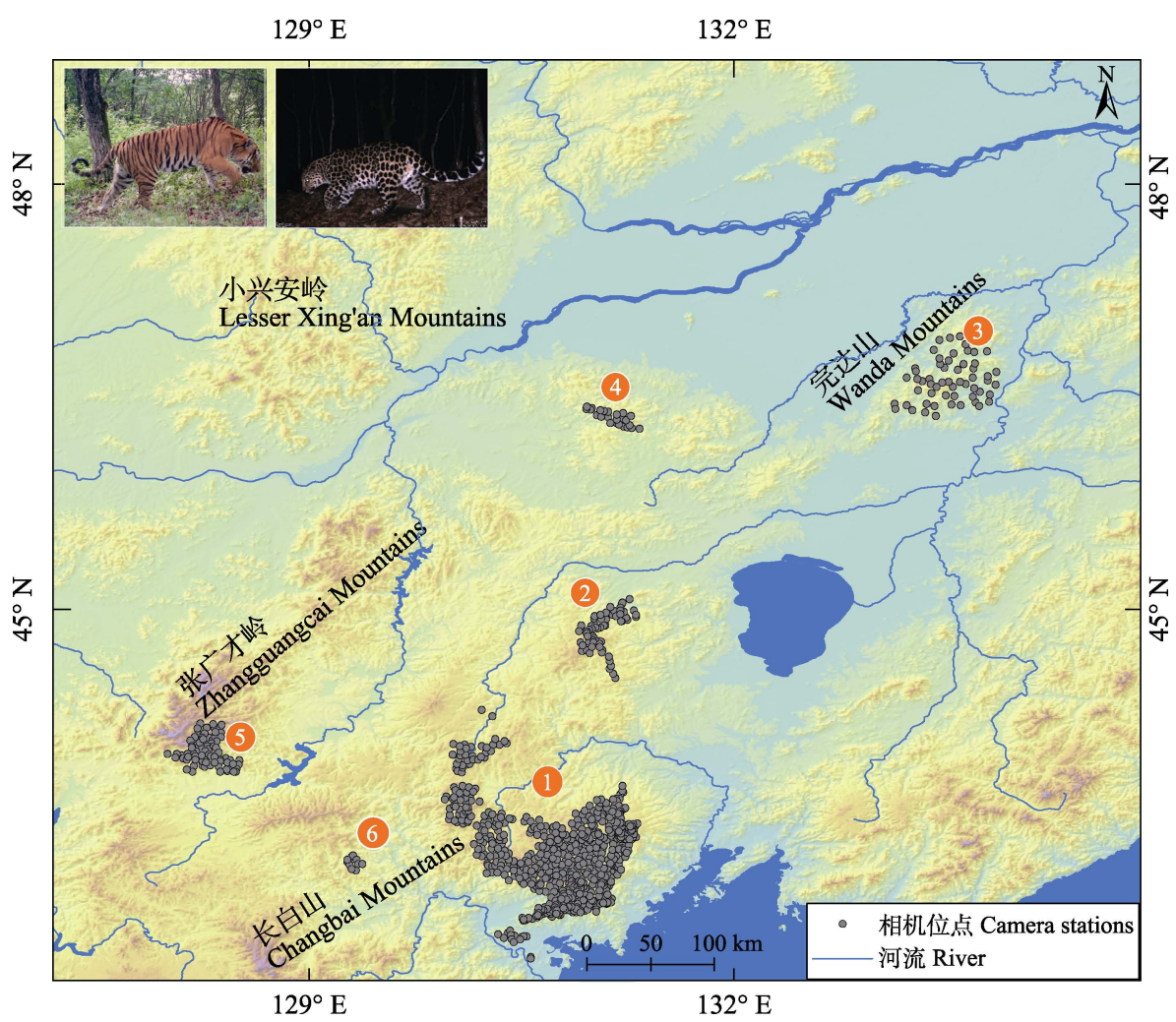

图1＼cjkstart东北虎豹生物多样性红外相机监测平台相机位点分布图。左上角的照片是分别于2007和2010年红外相机拍摄的东北虎 和东北豹个体。

Fig. 1 Map of the Long-term Tiger-Leopard Observation Network based on camera traps in Northeast China, with the insets showing two wild Amur tiger and leopard individuals photographed in China in 2007 and 2010, respectively.

scrofa)、梅花鹿 (Cervus nippon) 和狍 (Capreolus pygargus) 的家域面积，将监测区按照 $3.6 \mathrm{~km} \times$ $3.6 \mathrm{~km}$ 划分成单元网格。如果森林覆盖率达到 $90 \%$ 以上，就在网格中设置至少 1 台红外相机，相机间 平均距离 $2.36 \mathrm{~km}$ 。另外, 为了提高虎豹个体识别和 探测率，在东北虎豹国家公园东部虎豹核心分布区 (面积约5,400 $\mathrm{km}^{2}$ )约70\%的位点设2台相机, 双向安 放, 并在部分区域进行了相机补充, 相机间距离大 约1 km。2006-2019年, 我们在监测区内共设置了 910 个相机位点, 架设的地点通常选择动物最可能 出现的地方, 包括兽道、山脊、土路、标记树、洞 穴和补盐点等。相机安装在乔木树干上, 离地面高 度0.4-0.8 m左右, 相机的镜头尽量顺着通道方向放 置, 避免阳光直射, 清除镜头前的杂物和小灌木等 遮挡物以保证最佳的角度拍摄动物, 相机处不放置 任何诱饵。使用的相机型号为猎科Ltl-6210被动式 红外触发相机, 相机设置为视频模式, 录像长度为 $15 \mathrm{~s}$, 拍摄间隔为 $1 \mathrm{~min}$, 全天 $24 \mathrm{~h} 工$ 作, 敏感度设 为低或中。所有相机加装铁壳和锁链以防被盗。由
于研究区域交通不便，每隔3-4个月检查电池状态 和更换数据存储卡。

(2)数据处理和建库。首先将视频初步整理, 删 除空拍的视频(主要是没有任何动物的视频), 然后 鉴定有效视频中出现的野生动物、人类活动(包括人 和车辆)和家养动物(牛、羊、马、狗和猫等)。随着 分析技术发展，2018年之后的视频数据首先通过人 工智能进行处理，然后再人工校正。考虑到同一动 物或人类活动在同一相机点短期内可能重复拍摄, 我们对视频进行独立事件判断，判断标准为：(i)相 同或不同物种的不同个体或车辆的连续视频; (ii)相 同物种或车辆的连续视频时间间隔大于 $30 \mathrm{~min}$; (iii) 相同物种或车辆的不连续视频。符合以上任意一条 即被定义为一次独立事件。确定独立事件后，将以 上数据导入到Access数据库, 该数据库记录了每个 视频拍摄的日期、时间、物种名、地理位置等信息。

目前，我们已经构建东北温带针阔混交林区哺 乳动物物种多样性数据库, 包括野生东北虎和东北 豹个体识别数据库、足迹图片数据库、粪便样品数 
据库、栖息地(生境)数据库。另外, 2015年, 我们 与俄罗斯豹地国家公园开展了中俄跨境东北虎豹 联合监测与研究, 双方签署合作协议, 建立了东北 虎和东北豹种群的联合数据库(Feng et al, 2017; Vitkalova et al, 2018)。

\section{3 数据量和物种名录}

自2006年7月至2019年6月，东北虎豹生物多样 性长期定位监测平台共建立6个监测点(图1), 覆盖 了长白山支脉老爷岭、张广才岭和完达山(表1), 累 计投入相机 4,000 余台, 有效相机工作日173.6万多 天, 产生视频记录超过78.5万条。目前每年产生红 外相机监测数据 $4 \mathrm{~T}$ 。

红外相机拍摄到 28 种野生兽类，隶属 5 目 12 科 (附录1)，其中食肉目 4 科 13 种，偶蹄目3科6种，啮齿 目 3 科 7 种, 兔形目 1 科 1 种, 劳亚食虫目 1 科 1 种。在 记录到的野生兽类中, 国家I级重点保护野生动物 有东北虎、东北豹、紫貂(Martes zibellina)、原鹰 (Moschus moschiferus) 和梅花鹿 5种; II 级有猞猁 (Lynx lynx)、棕熊(Ursus arctos)、黑熊(U. thibetanus)、 黄喉貂(Martes flavigula)、水獭(Lutra lutra)、马鹿 (Cervus elaphus)和獐(Hydropotes inermis) 7种, 獐 是近20年来在东北地区重新发现。东北豹被IUCN 红色物种名录(http://www.iucnredlist.org)列为极危
物种(CR), 东北虎被列为濒危物种(EN), 黑熊、原 鸲和獐被列为易危物种 $(\mathrm{VU})$ 、水獭列为近危物种 $(\mathrm{NT})$ 。东北虎、东北豹、原謝和梅花鹿被《中国脊 椎动物红色名录》(蒋志刚等, 2016)列为极危物种; 猞猁、水獭和东北马鹿被列为濒危物种; 豹猫 (Prionailurus bengalensis)、黑熊和棕熊等6种被列为 易危物种。

红外相机拍摄到 32 种野生鸟类，隶属 8 目 15 科, 其中鸡形目 1 科 2 种，雁形目 1 科 2 种，鸽形目 1 科 1 种, 行形目 1 科 1 种，鹰形目 1 科 5 种，鸮形目 1 科 1 种，豚 木鸟目1科3种，雀形目8科17种。在记录到的野生鸟 类中, 国家I级重点保护野生动物有白尾海雕 (Haliaeetus albicilla), II 级有花尾榛鸡 (Tetrastes bonasia)、鹗春 (Aix galericulata)、秃鹑(Aegypius monachus)、松雀鹰(Accipiter virgatus)、苍鹰 (A. gentilis)、普通羔 (Buteo buteo)和长尾林鸮 (Strix uralensis) 7种。秃熟被IUCN红色物种名录列为近危 物种(NT)。白尾海雕被《中国脊椎动物红色名录》 列为易危物种，驾春、秃熟、苍鹰和长尾林鸮被列 为近危物种。

\section{4 主要发现和贡献}

目前，我们通过东北虎豹生物多样性红外相机 监测平台调查和分析了野生东北虎豹在中国境内

表1 东北虎豹生物多样性红外相机监测平台内各监测点基本信息列表

Table 1 Basic information of each monitoring sites across the Long-term Tiger-Leopard Observation Network

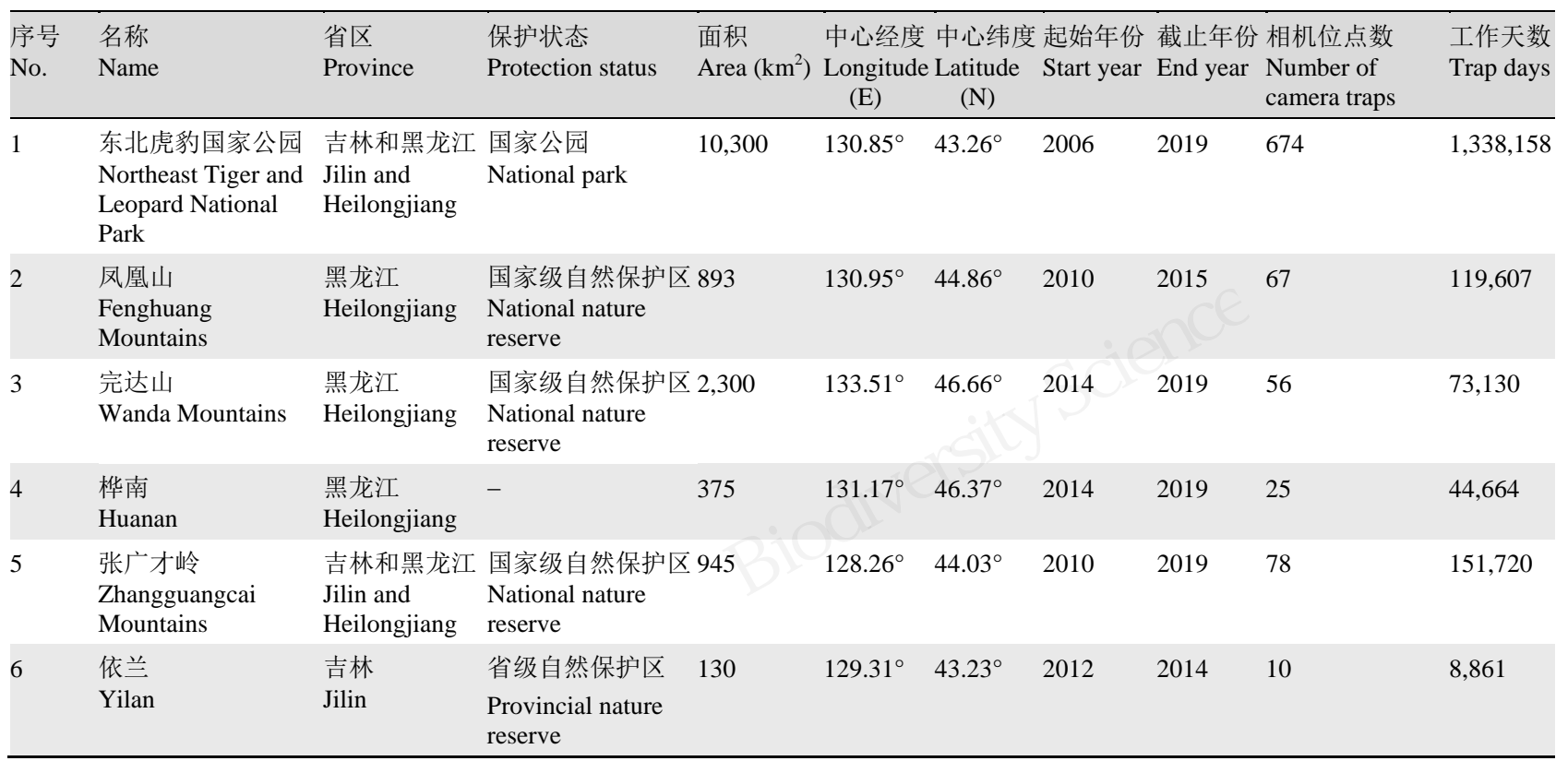


的种群数量、分布、密度和跨境活动规律, 系统评 价了人类干扰特别是放牧活动对东北虎豹及其猎 物多度、分布、行为和扩散的影响; 分析了东北虎 豹的食性构成和偏好, 并通过分析虎豹与其主要猎 物梅花鹿、野猪和狍子的时空重叠, 阐明了它们的 捕食策略; 从时间、空间和食物资源三个维度上分 析了东北虎和东北豹的竞争与共存机制; 分析了小 型和大型食肉动物在人为干扰景观下的时空作用 关系，进一步推动了对物种区域共存机制的理解。 2015年我们完成的《关于实施中国野生东北虎、豹 恢复与保护重大生态工程的建议》得到了国家领导 人的重要批示, 相关建议列入了十三五规划, 推动 了中国东北虎豹国家公园体制试点建设，相关成果 在“改革开放 40 周年成就展”上展出; 同年完成的 《虎豹回归中国计划的建议》被吉林省政府采纳。 2017年完成的《东北虎豹国家公园自然资源监测标 准》被国家林业和草原局采纳。有关东北虎和东北 豹的研究成果被Science 杂志专题报道, 发表在 Landscape Ecology杂志的虎豹研究论文(Wang et al, 2016)入选2017年Springer Nature集团发布的“可以 改变世界的180篇年度杰出论文”。重要发现如下。

\section{1 东北虎和东北豹种群数量和密度估计}

监测平台于 2007年6月以及2010年10月分别拍 摄到我国第一张自然状态下东北虎以及东北豹的 活动照片, 证明了我国境内仍然有野生东北虎豹活 动(Feng, 2011)。监测平台于2013年11月在距离中俄 边界20 $\mathrm{km}$ 的吉林珲春腹地拍摄到1只雌性东北虎 携带4只幼崽活动的影像资料, 2014年监测到1只雄 性东北虎从俄罗斯豹地国家公园向我国腹地迁移 的全过程(Wang et al, 2014, 2015), 表明了东北虎种 群向我国内陆扩散的趋势。2012年8月至2014年7月 期间, 在我国境内共监测到至少26只东北虎和42只 东北豹, 并记录了部分个体从成功繁殖到子代成年, 然后扩散定居的过程(Wang et al, 2016)。我们与俄罗 斯豹地公园监测数据联合分析, 表明2014年在约 $9,000 \mathrm{~km}^{2}$ 的中俄边境区域至少存在87只东北豹个 体(36只成年雌性, 34只成年雄性, 8只未知性别成体 以及 9 只亚成体), 其中有 31 只豹个体跨境活动; 另 外, 在该区域还同期分布着至少38只东北虎个体, 其中至少14只虎拥有“双国籍” (Feng et al, 2017)。空 间捕获-再捕获模型(SECR)显示中国境内东北虎密 度为 $0.20-0.27$ 只 $/ 100 \mathrm{~km}^{2}$ (Xiao et al, 2016; Wang et al, 2018); 东北豹密度为 $0.30-0.42$ 只 $/ 100 \mathrm{~km}^{2}$, 显著 低于俄罗斯种群的密度(大约1.40只 $/ 100 \mathrm{~km}^{2}$ )。中俄 联合监测数据进一步表明部分在中国拍摄的东北 豹，其活动中心在俄罗斯 (Wang et al, 2017; Vitkalova et al, 2018)。总之, 中俄跨境合作研究首 次完成了东北豹种群和东北虎1个小种群的生存状 态评估, 为这两个濒危物种的跨境保护提供了重要 的科学基础。

\section{2 人类活动对东北虎豹扩散的影响}

监测数据表明东北虎豹种群尽管有明显向我 国内陆扩散的趋势, 但大多数个体主要聚集于中俄 边境附近，林下放牧等各种人类干扰导致猎物短缺， 严重制约了虎、豹种群向中国内陆的扩散和定居(肖 文宏等, 2014; Wang et al, 2016)。研究表明, 东北虎 的生境利用远离高放牧区、居民点和主要道路, 随 着梅花鹿多度和森林覆盖率的增加而增加(Wang et al, 2018; Xiao et al, 2018; Yang et al, 2019)。同样，东 北豹的生境利用与猎物的多样性显著相关, 其次豹 避开道路和居民点, 特别是避开放牧地区(Wang et $\mathrm{al}, 2017)$ 。总之, 牛的长期放牧活动已导致猎物生境 退化, 尤其是牛替代了虎豹最主要的猎物梅花鹿 (Feng et al, 2018), 导致虎和豹在长期放牧地区广泛 缺失。该研究在猎物恢复、减少人类干扰等方面提 出了具体的建议, 特别强调逐步减少森林中的放牧 活动和人类干扰, 扩大梅花鹿的分布范围和增加种 群数量应是优先的保护行动。

\section{3 东北虎豹食性与捕食策略分析}

为了准确获知中国境内东北虎和东北豹的食 性，我们应用粪便分析法对采集的虎豹粪便内容物 进行分析, 确定其食物中猎物组成。同时, 结合红 外相机技术估计环境中猎物种群多度, 确定食性偏 好(Yang et al, 2018a; Dou et al, 2019)。另外, 我们还 评估了东北虎与猎物的时空重叠情况, 进一步解释 东北虎的食物选择机制。研究表明尽管东北虎豹食 性存在不同的季节性变化，但野猪、梅花鹿和狍是 对其生物量贡献率最高的猎物(占到 $75 \%-80 \%$ 以 上)。东北虎豹的食物中也包括了家养动物狗和牛, 这加剧了人兽冲突和疾病传播(如犬瘟热)的风险 (Soh et al, 2014; Wang et al, 2016; Sulikhan et al, 2018)。食性偏好分析表明东北虎极度偏好捕食野猪, 其次为梅花鹿，对狍无明显偏好，而东北豹偏好 狍。东北虎及其主要猎物的时空重叠分析结果显示, 
尽管梅花鹿与东北虎空间重叠度较高, 但两者活动 高峰明显错开; 而野猪与东北虎虽然空间重叠度较 低, 但两者活动高峰明显一致。结合食性分析结果, 我们认为东北虎与梅花鹿和野猪的这种时空分布 模式是其捕食策略的一种权衡(Dou et al, 2019)。

\section{4 东北虎豹竞争与共存}

大型食肉动物种间竞争与共存是保护生物学 的核心科学问题。我们应用大尺度红外相机监测数 据和野外生境调查数据, 首次从空间生态位和时间 生态位等方面探究了东北虎豹在人为干扰以及猎 物资源驱动下的竞争与共存机制(Yang et al, 2018b; Li et al, 2019)。双物种占域模型结果表明，东北虎豹 在空间上表现出独立的占域关系, 并且东北豹广泛 利用高海拔和山脊, 虎则主要出现在低海拔和频繁 利用林中土路, 进一步促进了二者的共存(Yang et al, 2019)。时间生态位分析表明，东北虎表现为夜行 性以及晨昏活动的节律, 而东北豹则以昼行性活动 为主, 因此时间生态位分化是促进东北虎豹景观共 存的重要因素(Li et al, 2019)。同时, 时空相互作用 分析结果也进一步证实了东北虎豹在时空生态位 上的分化。东北虎与人类活动表现出较高的空间生 态位重叠, 但是东北虎白天活动较少, 以此对人类 活动产生时间上的规避(Xiao et al, 2018), 东北豹则 在空间上明显避开人类活动。放牧活动严重限制了 东北虎豹和主要猎物的空间利用, 并对东北豹产生 较大的空间排除作用。总之, 有限的生境面积, 以 及这 2 种大型猫科动物对主要有蹄类猎物的竞争 (Yang et al, 2018a), 可能加剧二者之间的竞争。

综上所述，东北虎豹的共存与竞争机制受到种 间干涉性竞争、猎物资源可获得性、人类以及放牧 活动等多重因素影响。

\section{5 后续工作计划和重点}

目前，该平台已集成新技术创新体系，依托中 国广播电视有线网和 $700 \mathrm{Mhz}$ 无线 $4 \mathrm{G}$ 网络, 在东北 虎豹国家公园内实现了红外相机监测数据的实时 传输、云端存储、在线访问和人工智能识别。平台 未来将针对野生动物监测需求, 研发实时智能红外 相机、动物定位项圈、声音传感器等野生动物智能 感知设备, 研发野生动物个体形态与声音智能识别 技术，建设新一代的“物联网 + 生态”监测系统, 应用现代技术开展长期、系统、网格化定点科学观
测, 获取食物链、生境、环境要素和干扰等原始资 料和基础数据。为了满足中国定量评估野生动物保 护状态的需求, 红外相机数据将根据研究需要在不 同机构间实现共享。平台未来将重点开展主要食肉 和食草动物的种群动态、受食、生境选择、种群 遗传学和行为生态学等方面研究, 探索生态系统 中食物链、食物网的动态及物种之间的相互作用 关系，以及多尺度生境丧失、破碎化和退化等对 关键动物物种生存的影响。综合研究物种濒危和 种群衰退的遗传学机制、动物濒危的生态学过程及 其保护对策。将持续为重要物种的跨境保护、景观 规划和东北虎豹国家公园的建设提供科学支撑，以 促进不同机构之间以及与外部保护团体之间的数 据共享。

\section{参考文献}

Dinerstein E, Loucks C, Wikramanayake E, Ginsberg J, Sanderson E, Seidensticker J, Forrest J, Bryja G, Heydlauff A, Klenzendorf S, Leimgruber P, Mills J, O’Brien TG, Shrestha M, Simons R, Songer M (2007) The fate of wild tigers. BioScience, 57, 508-514.

Dou HL, Yang HT, Smith JLD, Feng LM, Wang TM, Ge JP (2019) Prey selection of Amur tigers in relation to the spatiotemporal overlap with prey across the Sino-Russian border. Wildlife Biology, 1, 1-11.

Feng LM, Shevtsova E, Vitkalova A, Matyukhina DS, Miquelle DG, Aramilev VV, Wang TM, Mou P, Xu RM, Ge JP (2017) Collaboration brings hope for the last Amur leopards. Cat News, 65, 20.

Feng LM, Wang TM, Mou P, Kou XJ, Ge JP (2011) First image of an Amur leopard recorded in China. Cat News, 55, 9.

Feng RN, Lu XY, Wang TM, Feng JW, Sun YF, Guan Y, Xiao WH, Smith JLD, Ge JP (2018) Effects of free-ranging livestock on sympatric herbivores at fine spatiotemporal scales. arXiv:1810.11192.

Hebblewhite M, Miguelle DG, Murzin AA, Aramilev VV, Pikunov DG (2011) Predicting potential habitat and population size for reintroduction of the Far Eastern leopards in the Russian Far East. Biological Conservation, 144, 2403-2413.

Henry P, Miquelle D, Sugimoto T, McCullough DR, Caccone A, Russello MA (2009) In situ population structure and ex situ representation of the endangered Amur tiger. Molecular Ecology, 18, 3173-3184.

Jiang ZG, Jiang JP, Wang YZ, Zhang E, Zhang YY, Li LL, Xie F, Cai B, Cao L, Zheng GM, Dong L, Zhang ZW, Ding P, Luo ZH, Ding CQ, Ma ZJ, Tang SH, Cao WX, Li CW, Hu HJ, Ma Y, Wu Y, Wang YX, Zhou KY, Liu SY, Chen YY, 
Li JT, Feng ZJ, Wang Y, Wang B, Li C, Song XL, Cai L, Zang CX, Zeng Y, Meng ZB, Fang HX, Ping XG (2016) Red List of China's Vertebrates. Biodiversity Science, 24, 500-551. (in Chinese and in English) [蒋志刚, 江建平, 王 跃招, 张鹗, 张雁云, 李立立, 谢锋, 蔡波, 曹亮, 郑光 美, 董路, 张正旺, 丁平, 罗振华, 丁长青, 马志军, 汤宋 华, 曹文宣, 李春旺, 胡慧建, 马勇, 吴毅, 王应祥, 周开 亚, 刘少英, 陈跃英, 李家堂, 冯祚建, 王燕, 王斌, 李 成, 宋雪琳, 蔡蕾, 蔵春金金, 曾岩, 孟智斌, 方红霞, 平晓 鸽 (2016) 中国脊椎动物红色名录. 生物多样性, 24, 500-551.]

Kelt DA, Heske EJ, Lambin X, Oli MK, Orrock JL, Ozgul A, Pauli JN, Prugh LR, Sollmann R, Sommer S (2019) Advances in population ecology and species interactions in mammals. Journal of Mammalogy, 100, 965-1007.

Li ZL, Wang TM, Smith JLD, Feng RN, Feng LM, Mou P, Ge JP (2019) Coexistence of two sympatric flagship carnivores in the human-dominated forest landscapes of Northeast Asia. Landscape Ecology, 34, 291-305.

Li ZW, Wu JG, Kou XJ, Tian Y, Wang TM, Mou P, Ge JP (2009) Land use pattern and its dynamic changes in Amur tiger distribution region. Chinese Journal of Applied Ecology, 20, 713-724. (in Chinese with English abstract) [李钟汶, 邬建国, 寇晓军, 田瑜, 王天明, 牟溥, 葛剑平 (2009) 东北虎分布区土地利用格局与动态. 应用生态学 报, 20, 713-724.]

Miquelle DG, Goodrich JM, Kerley LL, Pikunov DG, Dunishenko YM, Aramiliev VV, Nikolaev IG, Smirnov EN, Salkina GP, Endi Z, Seryodkin IV, Carroll C, Gapanov VV, Fomenko PV, Kostyria AV, Murzin AA, Quigley HB, Hornocker MG (2010a) Science-based conservation of Amur tigers in the Russian Far East and Northeast China. In: Tiger of the World: The Science, Politics, and Conservation of Panthera tigris, 2nd edn. (eds Tilson R, Nyhus PJ), pp. 403-423. Academic Press, Oxford.

Miquelle DG, Goodrich JM, Smirnov EN, Stephens PA, Zaumyslova OY, Chapron G, Kerley L, Murzin AA, Hornocker MG, Quigley HB (2010b) Amur tiger: A case study of living on the edge. In: Biology and Conservation of Wild Felids (eds Macdonald D, Loveridge A), pp. 325-339. Oxford University Press, Oxford.

Pikunov DG, Miquelle DG, Abramov VK, Nikolaev IG, Seredkin IV, Murzin AA, Korkishko VG (2003) A Survey of Far Eastern Leopard and Amur Tiger Populations in Southwest Primorski Krai, Russian Far East (February 2003). Pacific Institute of Geography (FEB RAS), Wildlife Conservation Society, and Tigris Foundation, Vladivostok.

Soh YH, Carrasco LR, Miquelle DG, Jiang JS, Yang J, Stokes EJ, Tang JR, Kang AL, Liu PQ, Rao M (2014) Spatial correlates of livestock depredation by Amur tigers in Hunchun, China: Relevance of prey density and implications for protected area management. Biological Conservation, 169, 117-127.
Sugimoto T, Aramilev VV, Kerley LL, Nagata J, Miquelle DG, McCullough DR (2014) Noninvasive genetic analyses for estimating population size and genetic diversity of the remaining Far Eastern leopard (Panthera pardus orientalis) population. Conservation Genetics, 15, 521-532.

Sulikhan NS, Gilbert M, Blidchenko EY, Naidenko SV, Ivanchuk GV, Gorpenchenko TY, Alshinetskiy MV, Shevtsova EI, Goodrich JM, Lewis JCM, Goncharuk MS, Uphyrkina OV, Rozhnov VV, Shedko SV, McAloose D, Miquelle DG, Seimon TA (2018) Canine distemper virus in a wild Far Eastern leopard (Panthera pardus orientalis). Journal of Wildlife Diseases, 54, 170-174.

Sunquist M (2010) What is a tiger? Ecology and behavior. In: Tiger of the World: The Science, Politics, and Conservation of Panthera tigris, 2nd edn. (eds Tilson R, Nyhus PJ), pp. 19-33. Academic Press, Oxford.

Tian Y, Wu JG, Kou XJ, Li ZW, Wang TM, Mou P, Ge JP (2009) Spatiotemporal pattern and major causes of the Amur tiger population dynamics. Biodiversity Science, 17, 211-225. (in Chinese with English abstract) [田瑜, 乌建国, 寇晓军, 李钟汶, 王天明, 牟溥, 葛剑平 (2009) 东北虎 种群的时空动态及其原因分析. 生物多样性, 17, 211-225.]

Vitkalova AV, Feng LM, Rybin AN, Gerber BD, Miquelle DG, Wang TM, Yang HT, Shevtsova EI, Aramilev VV, Ge JP (2018) Transboundary cooperation improves endangered species monitoring and conservation actions: A case study of the global population of Amur leopards. Conservation Letters, 11, e12574.

Walston J, Robinson JG, Bennett EL, Breitenmoser U, da Fonseca GAB, Goodrich J, Gumal M, Hunter L, Johnson A, Karanth KU, Leader-Williams N, MacKinnon K, Miquelle D, Pattanavibool A, Poole C, Rabinowitz A, Smith JLD, Stokes EJ, Stuart SN, Vongkhamheng C, Wibisono H (2010) Bringing the tiger back from the brink-The six percent solution. PLoS Biology, 8, e1000485.

Wang TM, Feng LM, Mou P, Ge JP, Li C, Smith JLD (2015) Long-distance dispersal of an Amur tiger indicates potential to restore the North-east China/Russian Tiger Landscape. Oryx, 49, 578-579.

Wang TM, Feng LM, Mou P, Wu JG, Smith JLD, Xiao WH, Yang HT, Dou HL, Zhao XD, Cheng YC, Zhou B, Wu HY, Zhang L, Tian Y, Guo QX, Kou XJ, Han XM, Miquelle DG, Oliver CD, Xu RM, Ge JP (2016) Amur tigers and leopards returning to China: Direct evidence and a landscape conservation plan. Landscape Ecology, 31, 491-503.

Wang TM, Feng LM, Yang HT, Han BY, Zhao YH, Juan L, Lu XY, Zou L, Li T, Xiao WH, Mou P, Smith JLD, Ge JP (2017) A science-based approach to guide Amur leopard recovery in China. Biological Conservation, 210, 47-55.

Wang TM, Royle JA, Smith JLD, Zou L, Lu XY, Li T, Yang HT, Li ZL, Feng RN, Bian YJ, Feng LM, Ge JP (2018) Living on the edge: Opportunities for Amur tiger recovery 
in China. Biological Conservation, 217, 269-279.

Wang TM, Yang HT, Xiao WH, Feng LM, Mou P, Ge JP (2014) Camera traps reveal Amur tiger breeding in NE China. Cat News, 61, 18-19.

Xiao WH, Feng LM, Mou P, Miquelle DG, Hebblewhite M, Goldberg JF, Robinson HS, Zhao XD, Zhou B, Wang TM, Ge JP (2016) Estimating abundance and density of Amur tigers along the Sino-Russian border. Integrative Zoology, 11, 322-332.

Xiao WH, Feng LM, Zhao XD, Yang HT, Dou HL, Cheng YC, Mou P, Wang TM, Ge JP (2014) Distribution and abundance of Amur tiger, Amur leopard and their ungulate preys in Hunchun National Nature Reserve, Jilin. Biodiversity Science, 22, 717-724. (in Chinese with English abstract) [肖文宏, 冯利民, 赵小丹, 杨海涛, 㝒海龙, 程 艳超, 牟溥, 王天明, 葛剑平 (2014) 吉林珲春自然保护 区东北虎和东北豹及其有蹄类猎物的多度与分布. 生物 多样性, 22, 717-724.]

Xiao WH, Hebblewhite M, Robinson H, Feng LM, Zhou B, Mou P, Wang TM, Ge JP (2018) Relationships between humans and ungulate prey shape Amur tiger occurrence in a core protected area along the Sino-Russian border. Ecology and Evolution, 8, 11677-11693.

Yang HT, Dou HL, Baniya RK, Han SY, Guan Y, Xie B, Zhao GJ, Wang TM, Mou P, Feng LM, Ge JP (2018a) Seasonal food habits and prey selection of Amur tigers and Amur leopards in Northeast China. Scientific Reports, 8, 6930.

Yang HT, Han SY, Xie B, Mou P, Kou XJ, Wang TM, Ge JP, Feng L (2019) Do prey availability, human disturbance and habitat structure drive the daily activity patterns of Amur tigers (Panthera tigris altaica)? Journal of Zoology, 307, 131-140.

Yang HT, Zhao XD, Han BY, Wang TM, Mou P, Ge JP, Feng LM (2018b) Spatiotemporal patterns of Amur leopards in northeast China: Influence of tigers, prey, and humans. Mammalian Biology, 92, 120-128.

(责任编委: 李晟 责任编辑: 时意专)

\section{附录 Supplementary Material}

附录1 东北虎豹生物多样性红外相机监测平台所记录的物种名录

Appendix 1 List of mammals and birds recorded by the Long-term Tiger Leopard Observation Network based on camera traps in Northeast, China (Before June 2019)

http://www.biodiversity-science.net/fileup/PDF/2020139-1.pdf 
王天明, 冯利民, 杨海涛, 鲍蕾, 王红芳, 葛剑平 (2020) 东北虎豹生物多样性红外相机监测平台概述. 生 物多样性, 28 (9): 1059-1066. http://www.biodiversity-science.net/CN/10.17520/biods.2020139

附录 1 东北虎豹生物多样性红外相机监测平台物种名录。红色名录级别: CR, EN, VU, NT 和 LC 分别为极 危, 濒危, 易危, 近危和无危。*1, 东北虎豹国家公园; 2, 凤凰山; 3 , 完达山; 4, 桦南; 5, 张广才岭; 6 , 依兰。详细的每个物种区域出现情况见图 1 和表 1 。

Appendix 1 List of mammals and birds recorded by the Long-term Tiger-Leopard Observation Network (TLON) of camera traps in Northeast, China (Before June 2019). EN, Endangered; CR, Critically endangered; LC, Least concern; NT, Near threatened; VU, Vulnerable. 1, Northeast Tiger and Leopard National Park; 2, Fenghuang Mountains; 3, Wanda Mountains; 4, Huanan; 5, Zhangguangcai Mountains; 6, Yilan.

\begin{tabular}{|c|c|c|c|c|}
\hline $\begin{array}{l}\text { 物种 } \\
\text { Species }\end{array}$ & $\begin{array}{l}\text { 国家重点保护野生动 } \\
\text { 物名录 } \\
\text { China's Protection } \\
\text { Level }\end{array}$ & $\begin{array}{l}\text { IUCN 红色名录 } \\
\text { IUCN Red List }\end{array}$ & $\begin{array}{l}\text { 中国脊椎动物红色名录 } \\
\text { Red List of China's Vertebrates }\end{array}$ & $\begin{array}{l}\text { 出现区域 } * \\
\text { Occurrence } \\
\text { area }\end{array}$ \\
\hline
\end{tabular}

\begin{tabular}{|c|c|c|c|c|}
\hline \multicolumn{5}{|l|}{$\begin{array}{l}\text { 兽类 Mammals } \\
\text { (一)食肉目 Carnivora } \\
\text { (1)猫科 Felidae }\end{array}$} \\
\hline 1. 东北虎 Panthera tigris altaica & I & EN & $\mathrm{CR}$ & $1,3-5$ \\
\hline 2. 东北豹 Panthera pardus orientalis & I & $\mathrm{CR}$ & $\mathrm{CR}$ & 1 \\
\hline 3. 豹猫 Prionailurus bengalensis & & LC & $\mathrm{VU}$ & $1-5$ \\
\hline $\begin{array}{l}\text { 4. 猞猁 Lynx lynx } \\
\text { (2)犬科 Canidae }\end{array}$ & II & $\mathrm{LC}$ & EN & 1,3 \\
\hline 5. 赤狐 Vulpes vulpes & & LC & NT & $1-5$ \\
\hline $\begin{array}{l}\text { 6. 貉 Nyctereutes procyonoides } \\
\text { (3)熊科 Ursidae }\end{array}$ & & $\mathrm{LC}$ & NT & $1-5$ \\
\hline 7. 棕熊 Ursus arctos & II & $\mathrm{LC}$ & VU & 1 \\
\hline $\begin{array}{l}\text { 8. 黑熊 Ursus thibetanus } \\
\text { (4)鼠科 Mustelidae }\end{array}$ & II & VU & VU & $1-6$ \\
\hline 9. 黄喉貂 Martes flavigula & II & $\mathrm{LC}$ & NT & $1-6$ \\
\hline 10. 紫貂 Martes zibellina & I & $\mathrm{LC}$ & $\mathrm{VU}$ & $1-3$ \\
\hline 11. 黄鼣 Mustela sibirica & & $\mathrm{LC}$ & LC & $1-6$ \\
\hline 12. 亚洲狗獾 Meles leucurus & & $\mathrm{LC}$ & NT & $1-6$ \\
\hline $\begin{array}{l}\text { 13. 水獭 Lutra lutra } \\
\text { (二)偶蹄目 Artiodactyla } \\
\text { (5)猪科 Suidae }\end{array}$ & II & NT & EN & 1 \\
\hline $\begin{array}{l}\text { 14. 野猪 Sus scrofa } \\
\text { (6)瘪科 Moschidae }\end{array}$ & & $\mathrm{LC}$ & $\mathrm{LC}$ & $1-6$ \\
\hline $\begin{array}{l}\text { 15. 原噟 Moschus moschiferus } \\
\text { (7)鹿科 Cervidae }\end{array}$ & I & $\mathrm{VU}$ & $\mathrm{CR}$ & 1 \\
\hline 16. 梅花鹿 Cervus nippon & I & $\mathrm{LC}$ & $\mathrm{CR}$ & $1,5,6$ \\
\hline 17. 马鹿 Cervus elaphus & II & $\mathrm{LC}$ & $\mathrm{EN}$ & $1-5$ \\
\hline 18. 狍 Capreolus pygargus & & $\mathrm{LC}$ & NT & $1-6$ \\
\hline $\begin{array}{l}\text { 19. 獐 Hydropotes inermis } \\
\text { (三)啮齿目 Rodentia } \\
\text { (8)松鼠科 Sciuridae }\end{array}$ & II & $\mathrm{VU}$ & $\mathrm{VU}$ & 1 \\
\hline 20. 松鼠 Sciurus vulgaris & & $\mathrm{LC}$ & NT & $1-6$ \\
\hline 21. 小飞鼠 Pteromys volans & & $\mathrm{LC}$ & $\mathrm{VU}$ & $1-6$ \\
\hline $\begin{array}{l}\text { 22. 北花松鼠 Tamias sibiricus } \\
\text { (9)仓鼠科 Cricetidae }\end{array}$ & & $\mathrm{LC}$ & LC & $1-6$ \\
\hline 23. 棕背鼠 Myodes rufocanus & & $\mathrm{LC}$ & $\mathrm{LC}$ & $1-6$ \\
\hline $\begin{array}{l}\text { 24. 大仓鼠 Tscherskia triton } \\
\text { (10)鼠科 Muridae }\end{array}$ & & $\mathrm{LC}$ & LC & $1-6$ \\
\hline 25. 大林姬鼠 Apodemus peninsulae & & LC & $\mathrm{LC}$ & $1-6$ \\
\hline
\end{tabular}


王天明, 冯利民, 杨海涛, 鲍蕾, 王红芳, 葛剑平 (2020) 东北虎豹生物多样性红外相机监测平台概述. 生 物多样性, 28 (9): 1059-1066. http://www.biodiversity-science.net/CN/10.17520/biods.2020139

\begin{tabular}{|c|c|c|c|c|}
\hline $\begin{array}{l}\text { 物种 } \\
\text { Species }\end{array}$ & $\begin{array}{l}\text { 国家重点保护野生动 } \\
\text { 物名录 } \\
\text { China's Protection } \\
\text { Level }\end{array}$ & $\begin{array}{l}\text { IUCN 红色名录 } \\
\text { IUCN Red List }\end{array}$ & $\begin{array}{l}\text { 中国脊椎动物红色名录 } \\
\text { Red List of China's Vertebrates }\end{array}$ & $\begin{array}{l}\text { 出现区域 * } \\
\text { Occurrence } \\
\text { area }\end{array}$ \\
\hline $\begin{array}{l}\text { 26. 黑线姬鼠 Apodemus agrarius } \\
\text { (四)兔形目 Lagomorpha } \\
\text { (11)兔科 Leporidae }\end{array}$ & & $\mathrm{LC}$ & $\mathrm{LC}$ & $1-6$ \\
\hline $\begin{array}{l}\text { 27. 东北兔 Lepus mandshuricus } \\
\text { (五)劳亚食虫目 Lipotyphla } \\
\text { (12)猬科 Erinaceidae }\end{array}$ & & $\mathrm{LC}$ & $\mathrm{LC}$ & $1-6$ \\
\hline 28. 东北刺猬 Erinaceus amurensis & & LC & $\mathrm{LC}$ & $1-6$ \\
\hline
\end{tabular}

\section{鸟类 Birds}

(一)鸡形目 Galliformes

(1)雉科 Phasianidae

1. 花尾榛鸡 Tetrastes bonasia

II

LC

LC

1-6

2. 环颈雉 Phasianus colchicus

LC

LC

1-6

(二)雁形目 Anseriformes

(2)鸭科 Anatidae

3. 豆雁 Anser fabalis

LC

LC

1-5

4. 驾蓄 Aix galericulata

II

LC

NT

1-5

(三)鸽形目 Columbiformes

(3)鸠鸽科 Columbidae

5. 山斑鸠 Streptopelia orientalis

LC

LC

$1-6$

(四)微形目 Charadriiformes

(4)䂆科 Scolopacidae

6. 丘鹬 Scolopax rusticola

LC

LC

$1,3,4$

(五)鹰形目 Accipitriformes

(5)鹰科 Accipitridae

7. 秃熟 Aegypius monachus

NT

NT

1

8. 松雀鹰 Accipiter virgatus II

LC

LC

1-6

9. 苍鹰 Accipiter gentilis

LC

$1,3,4,5$

10. 白尾海雕 Haliaeetus albicilla I

LC

NT

1

11. 普通鵟 Buteo japonicus

LC

$\mathrm{VU}$

$1-5$

(六)鸮形目 Strigiformes

(6)鸱鸮科 Strigidae

12. 长尾林鸮 Strix uralensis

II

LC

NT

$1-5$

(七)豚木鸟目 Piciformes

(7)豚木鸟科 Picidae

13. 白背啄木鸟 Dendrocopos

LC

LC

$1-6$

leucotos

14. 大斑豚木乌 Dendrocopos major

LC

LC

1-5

15. 灰头绿豚木鸟 Picus canus

LC

LC

$1-5$

\section{(八)雀形目 Passeriformes}

(8)鸦科 Corvidae

16. 松鸦 Garrulus glandarius

LC

LC

1-6

17. 灰喜鹊 Cyanopica cyanus

LC

$\mathrm{LC}$

1-6

18. 小嘴乌鸦 Corvus corone

LC

LC

1-6

19. 大嘴乌鸦 Corvus macrorhynchos

LC

LC

$1-6$

(9)山雀科 Paridae 
王天明, 冯利民, 杨海涛, 鲍蕾, 王红芳, 葛剑平 (2020) 东北虎豹生物多样性红外相机监测平台概述. 生 物多样性, 28 (9): 1059-1066. http://www.biodiversity-science.net/CN/10.17520/biods.2020139

\begin{tabular}{|c|c|c|c|c|}
\hline $\begin{array}{l}\text { 物种 } \\
\text { Species }\end{array}$ & $\begin{array}{l}\text { 国家重点保护野生动 } \\
\text { 物名录 } \\
\text { China's Protection } \\
\text { Level }\end{array}$ & $\begin{array}{l}\text { IUCN 红色名录 } \\
\text { IUCN Red List }\end{array}$ & $\begin{array}{l}\text { 中国脊椎动物红色名录 } \\
\text { Red List of China's Vertebrates }\end{array}$ & $\begin{array}{l}\text { 出现区域 * } \\
\text { Occurrence } \\
\text { area }\end{array}$ \\
\hline 20. 沼泽山雀 Poecile palustris & & $\mathrm{LC}$ & LC & $1-5$ \\
\hline $\begin{array}{l}\text { 21. 大山雀 Parus cinereus } \\
\text { (10)鸩科 Sittidae }\end{array}$ & & $\mathrm{LC}$ & $\mathrm{LC}$ & $1-5$ \\
\hline $\begin{array}{l}\text { 22. 普通鸩 Sitta europaea } \\
\text { (11)鸫科 Turdidae }\end{array}$ & & $\mathrm{LC}$ & $\mathrm{LC}$ & $1-5$ \\
\hline 23. 白眉地冻 Zoothera sibirica & & $\mathrm{LC}$ & $\mathrm{LC}$ & $1-5$ \\
\hline 24. 虎斑地冻 Zoothera aurea & & $\mathrm{LC}$ & $\mathrm{LC}$ & 1 \\
\hline 25. 灰背冻 Turdus hortulorum & & $\mathrm{LC}$ & $\mathrm{LC}$ & $1-5$ \\
\hline 26. 白腹鸫 Turdus pallidus & & $\mathrm{LC}$ & $\mathrm{LC}$ & $1-6$ \\
\hline $\begin{array}{l}\text { 27. 斑冻 Turdus eunomus } \\
\text { (12)鹟科 Muscicapidae }\end{array}$ & & $\mathrm{LC}$ & $\mathrm{LC}$ & $1-5$ \\
\hline $\begin{array}{l}\text { 28. 红胁蓝尾鸲 Tarsiger cyanurus } \\
\text { (13)能鸰科 Motacillidae }\end{array}$ & & $\mathrm{LC}$ & $\mathrm{LC}$ & $1-6$ \\
\hline $\begin{array}{l}\text { 29. 灰能鸰 Motacilla cinerea } \\
\text { (14)燕雀科 Fringillidae }\end{array}$ & & $\mathrm{LC}$ & $\mathrm{LC}$ & $1-5$ \\
\hline 30. 黑尾蜡嘴雀 Eophona migratoria & & $\mathrm{LC}$ & $\mathrm{LC}$ & 1 \\
\hline $\begin{array}{l}\text { 31. 北朱雀 Carpodacus roseus } \\
\text { (15)鸥科 Emberizidae }\end{array}$ & & $\mathrm{LC}$ & LC & 1 \\
\hline 32. 灰头鸥 Emberiza spodocephala & & $\mathrm{LC}$ & $\mathrm{LC}$ & $1-6$ \\
\hline
\end{tabular}

